\title{
Variación espacial y temporal en la producción de gametangios en la macroalga exótica Codium fragile subsp. tomentosoides (Chlorophyta: Bryopsidales) en el submareal de Caldera, Chile
}

Spatial and temporal variation in the output of gametangia of the exotic macroalga Codium fragile subsp. tomentosoides (Chlorophyta: Bryopsidales) in the subtidal zone of Caldera, Chile

\section{Cristóbal Villaseñor-Parada ${ }^{1,2,3,4}$, Erasmo C. Macaya ${ }^{2}$, Luis M. Jara-Rojas ${ }^{5}$ y Paula E. Neill ${ }^{5}$}

\author{
${ }^{1}$ Departamento de Botánica, Facultad de Ciencias Naturales y Oceanográficas, Universidad de Concepción, Casilla 160-C, \\ Concepción, Chile. cvillasenor@udec.cl \\ ${ }^{2}$ Laboratorio de Estudios Algales (ALGALAB), Departamento de Oceanografía, Facultad de Ciencias Naturales y \\ Oceanográficas, Universidad de Concepción, Casilla 160-C, Concepción, Chile \\ ${ }^{3}$ Laboratorio de Invasiones Biológicas (LIB), Facultad de Ciencias Forestales, Universidad de Concepción, Casilla 160-C, \\ Concepción, Chile \\ ${ }^{4}$ Instituto de Ecología y Biodiversidad (IEB), Facultad de Ciencias, Universidad de Chile, Casilla 653, Santiago, Chile \\ ${ }^{5}$ Departamento de Ecología, Facultad de Ciencias, Universidad Católica de la Santísima Concepción, Casilla 297, Concepción, \\ Chile
}

\begin{abstract}
The macroalga Codium fragile ssp. tomentosoides is characterized by exclusive asexual reproduction through parthenogenesis. We describe the spatial (within thallus) and temporal (seasonal) variability in the abundance of reproductive structures (gametangia) in specimens of $C$. fragile collected from the subtidal zone of Caldera, Chile. Gametangia are more frequent on newer sections of the thallus (apical and middle), increasing during austral springsummer, and with thallus size (i.e., length and number of dichotomies). Given that apical fragments are highly buoyant and thallus fragmentation increases during the summer, drifting fragments may be important dispersal vectors contributing to the spread of this species to new sites.
\end{abstract}

Key words: Dispersal, fragmentation, invasion, propagule pressure

\section{INTRODUCCIÓN}

Codium fragile (Suringar) Hariot, es una macroalga verde originaria de Japón, reconocida mundialmente como invasora en diversos ecosistemas (Chapman 1999, Trowbridge 1998, 1999; Mathieson et al. 2003, Neill et al. 2006). En la subespecie tomentosoides, a diferencia del resto de las subespecies de $C$. fragile, sólo se han reportado gametos femeninos, por lo que la reproducción es exclusivamente asexual, mediante partenogénesis (Trowbridge 1998, Prince \& Trowbridge 2004), sugiriendo que esta macroalga estaría compuesta por unos pocos clones a nivel mundial (Trowbridge 1998, Provan et al. 2004); de aquí en adelante como $C$. fragile se hará referencia a la subespecie tomentosoides.

Debido a su reproducción estrictamente asexual, $C$. fragile presenta un ciclo de vida muy particular, en el cual los gametos, sin fusionarse, dan origen a una fase críptica inicial, en la que el talo está compuesto por filamentos microscópicos haploides (estado vaucherioide sensu Trowbridge 1998), los que posteriormente adquieren la morfología característica de dosel boyante (Trowbridge 1998, Prince \& Trowbridge 2004). Este dosel es de tipo sifonoso y está formado por numerosos filamentos entretejidos que componen las 2 regiones del talo: la médula (región central), compuesta por filamentos incoloros; y la corteza (región periférica), compuesta por utrículos, en cuyas paredes laterales se desarrollan estructuras reproductivas denominadas gametangios (Churchill \& Moeller 1972, Trowbridge 1998, Prince \& Trowbridge 2004).

No existe un claro patrón de distribución de gametangios en el talo de $C$. fragile, los que se encuentran a lo largo de todo el talo (Trowbridge 1998), presentando alta variabilidad entre individuos e incluso dentro de un mismo individuo (Bulleri et al. 2007). 
Estudios sobre la fenología reproductiva de $C$. fragile en la costa Atlántica de Estados Unidos muestran mayor producción de gametangios durante otoño-invierno, con posterior liberación de gametos a principios de primavera, y grandes diferencias en fecundidad entre sitios (Churchill \& Moeller 1972). Sin embargo, este patrón estacional en la subespecie no necesariamente se aplica a otras latitudes (Trowbridge 1998). De hecho, Bulleri et al. (2007) describen la fenología reproductiva en el mar Adriático, sin distinguir un máximo estacional, debido a la alta variabilidad en la producción de gametangios entre talos, aunque en esta zona se reconoce un ciclo anual de abundancia (Bulleri \& Airoldi 2005), tal como ocurre en otras localidades donde $C$. fragile ha sido introducida (Trowbridge 1999, Neill 2007).

En Chile, Codium fragile se distribuye desde el Norte Chico hasta el Extremo Austral de manera discontinua (Neill et al. 2006), siendo una de las pocas especies invasoras reportadas para esta costa (i.e., con expansión del rango desde su punto de introducción, sensu Richardson et al. 2000). En Caldera esta macroalga ha sido reportada en ambientes inter y submareales (González \& Santelices 2004, Provan et al. 2004, Neill et al. 2006, Villaseñor-Parada \& Neill 2011) afectando negativamente los cultivos de Gracilaria donde ha sido considerada 'peste' (Neill et al. 2006). Al igual que lo reportado para otras localidades (e.g., Trowbridge 1999, Bulleri \& Airoldi 2005), también se observa un marcado ciclo anual de abundancia, dominado por la morfología de dosel boyante (talo erecto) desde invierno hasta fines del verano; seguido de un periodo donde incrementa la morfología de filamentos no diferenciados (estado 'vaucherioide') hasta mediados de otoño; y finalmente un periodo entre finales de otoño y principios de invierno donde dominan talos erectos sin ramificaciones (Neill 2007).

Basado en lo anterior, los objetivos fundamentales de este trabajo fueron caracterizar patrones de distribución temporal (entre estaciones) y espacial (dentro del talo) de la abundancia de gametangios de $C$. fragile en la localidad de Caldera, y evaluar la relación entre la abundancia de gametangios y el tamaño del talo.

\section{Materiales Y MÉTODOS}

Se recolectaron aleatoriamente 19 ejemplares de Codium fragile, entre primavera 2004 e invierno 2005, desde Bahía Calderilla (2704'39”'S; 7050'70”'O) en Caldera, Chile. Los talos fueron extraídos desde la zona submareal mediante buceo autónomo. Cinco talos fueron recolectados durante primavera (octubre, noviembre), 4 durante verano (enero, febrero), 4 durante otoño (abril, mayo) y 6 durante invierno (julio, agosto), los cuales fueron trasladados al laboratorio para estimar el tamaño de cada talo mediante mediciones morfométricas de longitud total, número de dicotomías y máxima circunferencia del dosel.

Para determinar la variabilidad espacial y temporal de gametangios en $C$. fragile, los 19 talos recolectados fueron divididos en 3 secciones: basal, media y apical, obteniendo 3 fragmentos de $5 \mathrm{~cm}$ cada uno desde cada sección (Fig.1a). En cada fragmento se realizaron 10 preparaciones de aplastamiento (i.e., 'squash') (Fig.1b). En cada 'squash' se observó la presencia o ausencia de gametangios, estimando la abundancia de estas estructuras reproductivas mediante su frecuencia de ocurrencia en cada fragmento (e.g., si se observara la presencia de gametangios en 8 de los 10 'squash', la frecuencia de ocurrencia de gametangios sería 0,8 ). Mediante ANDEVA de dos vías se evaluó el efecto de la sección del talo (variabilidad espacial) y la estación del año de recolección (variabilidad temporal), sobre la frecuencia de gametangios. Para cumplir con supuestos de normalidad y homocedasticidad los datos fueron sometidos a transformación angular (i.e., arcoseno de la raíz cuadrada). Grupos homogéneos fueron identificados mediante el análisis a posteriori de Fisher.

Para determinar el efecto del tamaño del talo sobre la producción de gametangios, se relacionó cada estimador de tamaño (i.e., longitud total, número de dicotomías y máxima circunferencia del dosel), con la frecuencia de gametangios, esta vez calculada para todo el talo (e.g., en cada talo se observaron 90 'squash', por lo que si se observaran estructuras reproductivas en 25 de ellos, la frecuencia de gametangios sería 0,28 para ese talo). La evaluación estadística de la relación entre frecuencia de gametangios y estimadores del tamaño del talo fue realizada mediante regresiones lineales de mínimos cuadrados.

\section{RESUltados Y DiscusióN}

Los gametangios emergían desde la base de los utrículos (uno o 2 por utrículo), en los que se reconocieron 2 tipos: gametangios con $100 \%$ de gametos y gametangios con $75 \%$ de gametos (Fig.1c). Al interior de gametangios maduros se observaron gametos biflagelados de pigmentación verde oscura (Fig.1d), morfológicamente similares a los descritos en trabajos anteriores (Churchill \& Moeller 1972, Trowbridge 1998, Prince \& Trowbridge 2004). 

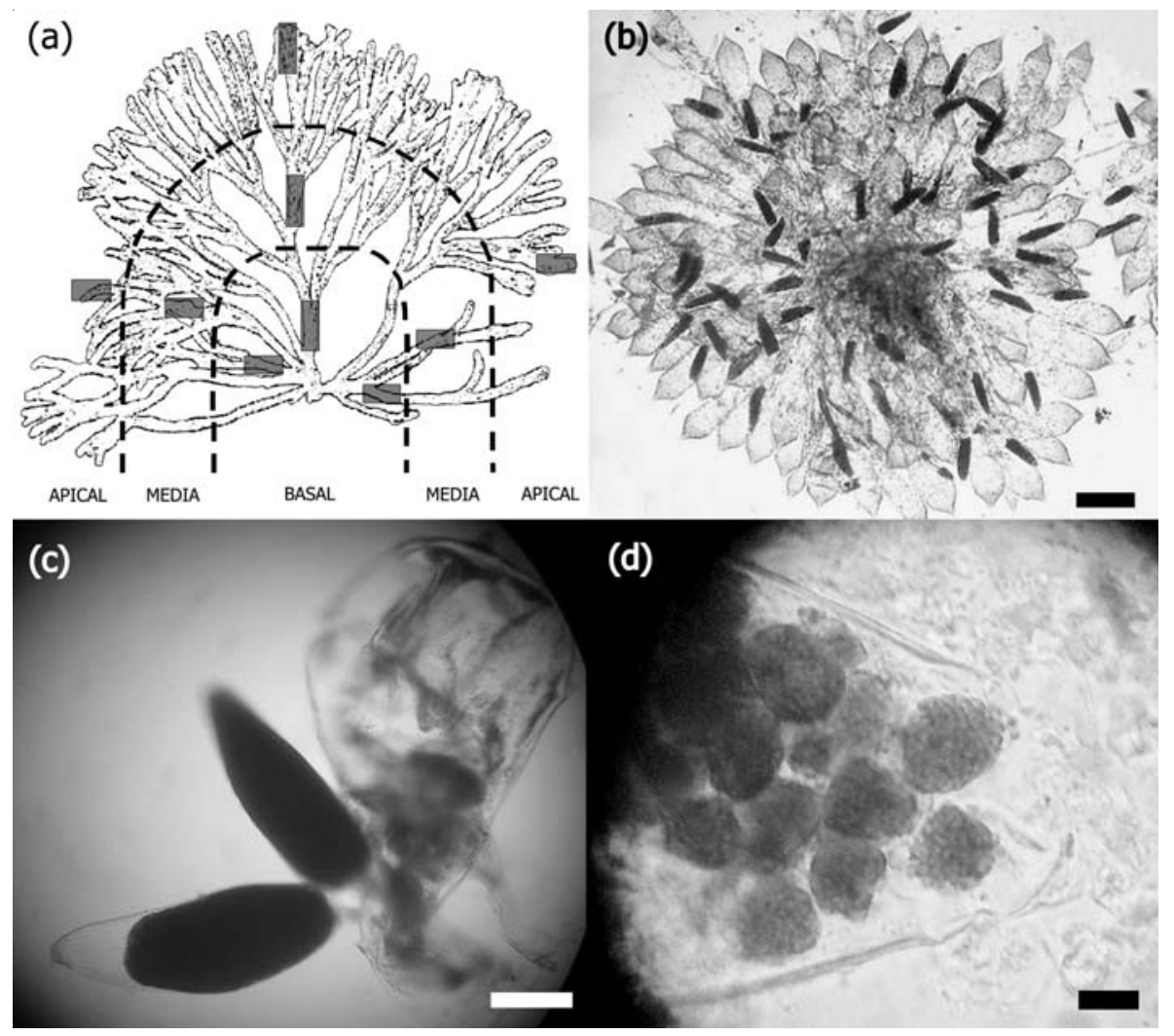

Figura 1. (a) Diseño experimental usado para obtener 9 fragmentos de cada uno de los 19 talos de Codium fragile en los cuales se determinó la frecuencia de gametangios (extraído de Villaseñor-Parada \& Neill 2011), (b) preparaciones permanentes o 'squash' de talos donde se observan los utrículos (estructuras claras) y gametangios (estructuras oscuras), (c) detalle de los gametangios alrededor de los utrículos; un gametangio con $100 \%$ de gametos y otro con $75 \%$, (d) detalle de los gametos en el interior de los gametangios. Barra indica en (b) $\mathbf{3 0 0} \mu \mathrm{m}$, (c) $100 \mu \mathrm{m}$ y (d) $10 \mu \mathrm{m} /$ (a) Experimental design used to obtain 9 fragments from each of the 19 Codium fragile thalli in which gamatengia frequency was determined (extracted from Villaseñor-Parada \& Neill 2011), (b) permanent or 'squash' preparations of thalli showing utricles (clear structures) and gametangia (dark structures), (c) detail of gametangia around the utricles; one gametangium with $100 \%$ and another with $75 \%$ of gametes, (d) detail of gametes within the gametangia. The scale bar indicates: (b) $300 \mu \mathrm{m}$, (c) $100 \mu \mathrm{m}$ and (d) $10 \mu \mathrm{m}$

Si bien se observó la presencia de gametangios en las 3 secciones del talo y a lo largo de todo el año, los resultados del ANDEVA de dos vías muestran una interacción significativa entre las secciones del talo y las estaciones (Fig. 2), apoyando la existencia de un patrón espacial (dentro del talo) y temporal (estacional) de gametangios en $C$. fragile de la localidad de Caldera. Entender la fenología reproductiva de esta especie invasora puede ser relevante para diseñar estrategias de manejo en el momento que se produzca una menor presión de propágulos.
La distribución de gametangios no fue uniforme a lo largo del talo, presentando una mayor frecuencia en las secciones más nuevas (i.e., apical y media), y con presencia ocasional en la sección basal (Fig. 2), que corresponde a la porción más vieja del talo (sensu Trowbridge 1998, 1999). La mayor cantidad de gametangios en la sección media respecto a la sección apical, particularmente en otoño e invierno, podría deberse a la ausencia de gametangios en las puntas de los ápices debido a un activo crecimiento (adición de utrículos) en este periodo, factor previamente descrito para esta subespecie (Trowbridge 1998). 


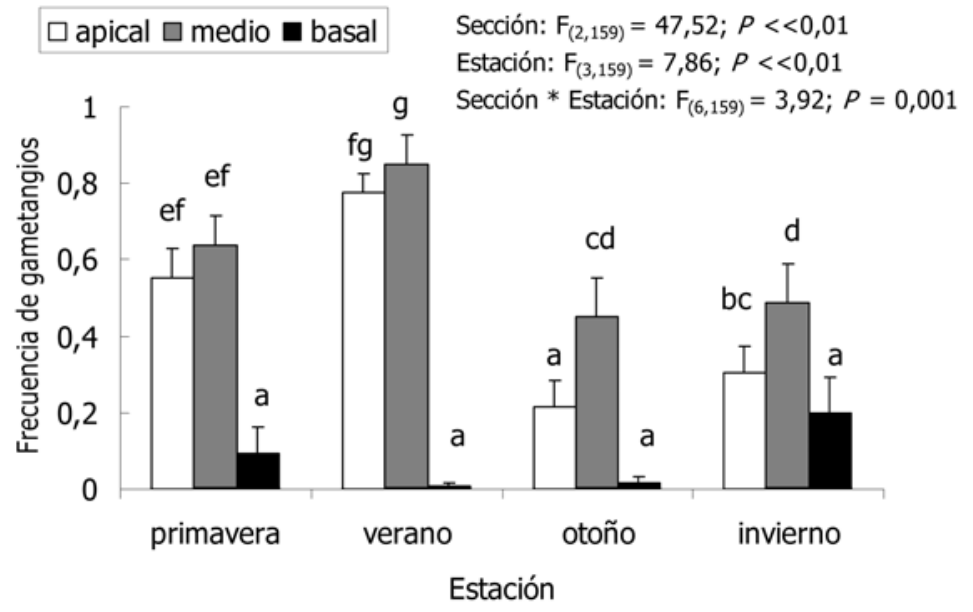

Figura 2. Frecuencia de gametangios (promedio + error estándar) en la sección apical, media y basal de talos de $C$. fragile recolectados durante primavera 2004 y verano, otoño e invierno 2005. Resultado del ANDEVA factorial se muestra sobre el gráfico, y grupos homogéneos detectados mediante la prueba a posteriori de Fisher se muestran con letras sobre las columnas / Frequency of gametangia (average + standard error) on the apical, middle and basal sections of $C$. fragile thalli collected during spring 2004 and summer, autumn and winter 2005 . The results of factorial ANOVA are shown on the graph, and homogeneous groups detected by the a posteriori Fisher test are indicated by letters above the bars. The graph shows untransformed data, while statistical analyses were conducted following angular transformation

El patrón espacial resultante sería relevante para entender la dispersión de esta especie dado que la gran cantidad de gametangios en las secciones apical y media corresponden también a la parte del talo con mayor flotabilidad (Gagnon et al. 2011). Así, fragmentos apicales a la deriva, podrían ser propágulos para la colonización de nuevos sitios. Fragmentos flotantes de macroalgas pueden sobrevivir y continuar creciendo una vez desprendidos del sustrato (Thiel \& Gutow 2005). Si éstos son capaces de liberar esporas o generar cigotos, podrían constituir un medio alternativo de dispersión, alcanzando mayores distancias que unidades de dispersión tradicionales (Macaya et al. 2005). De hecho, fragmentos de $C$. fragile a la deriva son considerados importantes vectores de introducción y expansión (Mathieson et al. 2003, Gagnon et al. 2011). En Chile, la expansión de $C$. fragile es asociada con la cercanía de otras poblaciones de C. fragile (Neill 2007), dado la mayor presión de propágulos generada por fragmentos a la deriva, los cuales podrían constituir importantes vectores de dispersión secundaria.

Aunque los gametangios están presentes durante todo el año, su frecuencia fue mayor en primavera-verano y menor en otoño-invierno (Fig. 2). Esto contrasta con poblaciones de la costa Atlántica de Estados Unidos, donde hay un incremento en la densidad de gametangios durante la época estival, y un máximo en otoño (Churchill \& Moeller 1972, Trowbridge 1998). Esta diferencia puede deberse a características propias de las localidades de estudio. Churchill \& Moeller (1972) concluyen que la producción de gametangios ocurre entre 12 y $15^{\circ} \mathrm{C}$, pero en Caldera la temperatura es mayor a $15^{\circ} \mathrm{C}$ durante primavera-verano $\left(16-19^{\circ} \mathrm{C}\right)$, y sólo es inferior a $15^{\circ} \mathrm{C}$ a fines del otoño y principios de invierno. Adicionalmente, en el mar Adriático los investigadores no reconocen máximos estacionales en la cantidad de gametangios, encontrando alta variabilidad entre talos, incluso dentro de un mismo sitio (Bulleri et al. 2007). En Caldera, la fragmentación de talos de $C$. fragile incrementa durante el verano (P. Neill, observ. pers.), tal como ocurre en otras localidades (Dromgoole 1975, Bégin \& Scheibling 2003). Además, en este periodo incrementa la frecuencia de gametangios en los talos y concuerda con una mayor presión de propágulos por fragmentos a la deriva.

Finalmente, se encontró una relación directa y positiva entre cantidad de gametangios y longitud (Fig. 3a), y número de dicotomías (Fig. 3b), lo que sugiere un incremento en la fecundidad con la edad del talo. En cambio, la máxima circunferencia del dosel no fue un buen predictor de la frecuencia de gametangios (Fig. 3c), aún cuando en otros trabajos (e.g., Bégin \& Scheibling 2003) si fue un buen estimador del tamaño. 

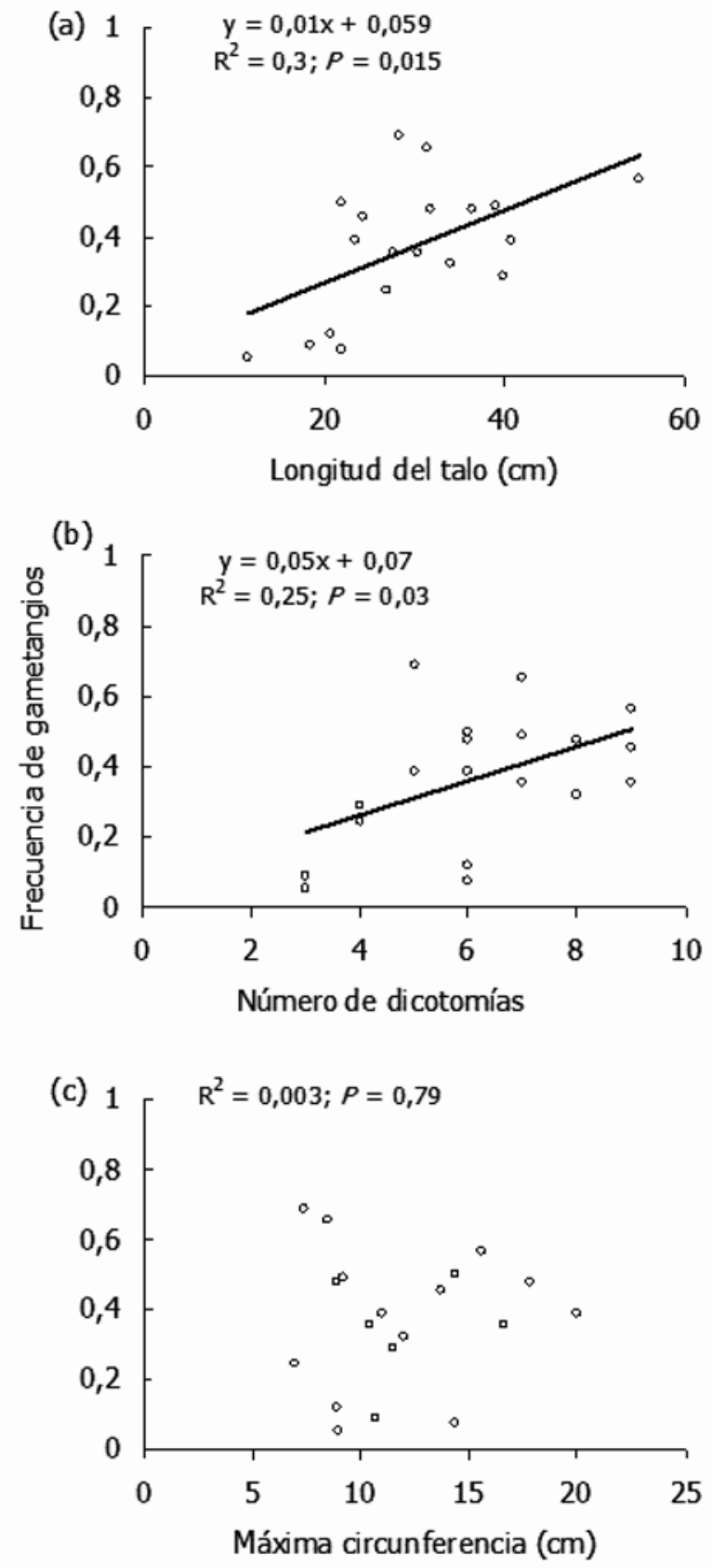

Figura 3. Relación entre la frecuencia de gametangios con: (a) longitud, (b) número de dicotomías y (c) máxima circunferencia de talos de $C$. fragile. Resultados de los análisis de regresión lineal se muestran para cada caso / Relationship between the frequency of gametangia with: (a) length, (b) number of dichotomies and (c) maximum circumference of $C$. fragile thalli. Results of linear regression analyses are provided for each case 
Gagnon KC, CW Mckindsey \& LE Johnson. 2011. Dispersal potential of invasive algae: the determinants of buoyancy in Codium fragile ssp. fragile. Marine Biology 158: 24492458.

González A \& B Santelices. 2004. A dichotomous species of Codium (Bryopsidales, Chlorophyta) is colonizing northern Chile. Revista Chilena de Historia Natural 77: 293-304.

Macaya EC, S Boltaña, IA Hinojosa, JE Macchiavello, NA Valdivia, NR Vásquez, AH Buschmann, JA Vásquez, JM Vega \& M Thiel. 2005. Presence of sporophylls in floating kelp rafts of Macrocystis spp. (Phaeophyceae) along the Chilean Pacific coast. Journal of Phycology 41: 913-922.

Mathieson AC, CJ Dawes, LG Harris \& EJ Hehre. 2003. Expansion of the Asiatic green alga Codium fragile subsp. tomentosoides in the Gulf of Maine. Rhodora 105(921): 153.

Neill PE. 2007. Distribución de la macroalga introducida Codium fragile (Chlorophyta) en Chile y sus efectos sobre la estructura de los ensambles del submareal rocoso del norte chico. Tesis Doctoral, Facultad de Ciencias Biológicas, Pontificia Universidad Católica de Chile, Santiago, 179 pp.

Neill PE, O Alcalde, S Faugeron, SA Navarrete \& JA Correa. 2006. Invasion of Codium fragile ssp. tomentosoides in northern Chile: a new threat for Gracilaria farming. Aquaculture 259: 202-210.
Prince JS \& CD Trowbridge. 2004. Reproduction in the green macroalga Codium (Chlorophyta): characterization of gametes. Botanica Marina 47: 461-470.

Provan J, S Murphy \& CA Maggs. 2004. Tracking the invasive history of the green alga Codium fragile ssp. tomentosoides. Molecular Ecology 14: 189-194.

Richardson DM, P Pysek, M Rejmánek, MG Barbour, FD Panetta \& CJ West. 2000. Naturalization and invasion of alien plants: concepts and definitions. Diversity and Distributions 6: 93-107.

Thiel M \& L Gutow. 2005. The ecology of rafting in the marine environment. II. The rafting organisms and community. Oceanography and Marine Biology: An Annual Review 43: 279-418.

Trowbridge CD. 1998. Ecology of the green macroalga Codium fragile (Suringar) Hariot 1889: invasive and non-invasive subspecies. Oceanography and Marine Biology: An Annual Review 36: 1-64.

Trowbridge CD. 1999. An assessment of the potential spread and options for control of the introduced green macroalga Codium fragile ssp. tomentosoides on Australia shores, 44 pp. Center for Research on Introduced Marine Pests, CSIRO, Marine Research, Hobart.

Villaseñor-Parada C \& PE Neill. 2011. Distribución espacial de epifitos en el talo de la macroalga introducida Codium fragile subsp. tomentosoides en el submareal de Caldera. Revista de Biología Marina y Oceanografía 46: 257-262.

Recibido el 12 de agosto de 2012 y aceptado el 31 de enero de 2013

Editor Asociado: Pilar Muñoz M. 